\title{
FIRST EIGENVALUE OF $p$-LAPLACIAN ALONG THE NORMALIZED RICCI FLOW ON BIANCHI CLASSES
}

\author{
Mohammad Javad Habibi Vosta $\operatorname{Kolaei}^{1}$, Shahroud Azami ${ }^{2}$ \\ ${ }^{1,2}$ Imam Khomeini international university, Department of pure \\ mathematics, Qazvin, Iran, \\ E-mail: mj.habibi@edu.ikiu.ac.ir, azami@sci.ikiu.ac.ir
}

\begin{abstract}
Consider $M$ as a 3 -homogeneous manifold. In this paper, we are going to study the behavior of the first eigenvalue of $p$-Laplace operator in a case of Bianchi classes along the normalized Ricci flow. Also we will give some upper and lower bounds for a such eigenvalue.

Key words and Phrases: Ricci flow, p-Laplace operator, Eigenvalue.
\end{abstract}

\section{INTRODUCTION}

Over the last few years, studying the geometric flows, specially the Ricci flow have become a topic of active research in both mathematics and physics. Generally, a geometric flow is an evolution of a geometric structure under a differential equation related to a functional usually associated with a curvature in a manifold. Although, the Ricci flow was introduced first by Hamilton [10] in mathematics and in the work of Friedan [9] in the context of string theory, Perelman has made a current wide interests by the proof of Poincare' conjecture using the Ricci flow in [16].

Consider $M$ as a manifold with Riemannian metric $g_{0}$, the family $g(t)$ of Riemannian metrics on $M$ have been called as an un-normalized Ricci flow when it satisfies the equation

$$
\frac{d}{d t} g(t)=-2 \operatorname{Ric}(g(t)) \quad g(0)=g_{0},
$$

2020 Mathematics Subject Classification: 53C45, 53C21, 58C40 Received: 08-06-2020, accepted: 11-10-2020. 
where Ric is known Ricci tensor of $g(t)$.

And also one can consider the normalized Ricci flow as follow

$$
\frac{d}{d t} g(t)=-2 \operatorname{Ric}(g(t))+\frac{2 r}{n} g \quad g(0)=g_{0},
$$

where $r=\frac{\int_{M} R d \mu}{\int_{M} d \mu}$ is the average of scalar curvature.

Hamilton in [10], has shown that there is a unique solution for the Ricci flow (1), on the interval $[0, T)$ for a sufficient $T$. Now consider $g(t)$ as a solution of the Ricci flow (1), the customary normalization on 3 -manifolds is setting

$$
\bar{g}(\bar{t})=\psi(t) g(t), \quad \bar{t}=\int_{0}^{t} \psi(\nu) d \nu,
$$

with $\frac{1}{\psi} \frac{\partial \psi}{\partial t}=\frac{2 r}{n}$ where $n=3$ and $r$ is as same as what mentioned above is average of scalar curvature. In this case $\bar{g}(\bar{t})$ will be the solution of normalized Ricci flow (2).

In [16], Perelman has shown that the first eigenvalue of $-\Delta+\frac{R}{4}$ is nondecreasing under the Ricci flow. Later Cao [4] has shown the similar result for the eigenvalues of $-\Delta+\frac{R}{2}$ on a manifolds with non-negative curvature operator. Also similar results hold for the first eigenvalue of $-\Delta+a R \quad\left(a \geq \frac{1}{4}\right)$ along the Ricci flow, for more details see $[5,14]$.

There are some other published work in monotonicity of eigenvalues of geometric operators under some geometric flows. For example second author in [2], has studied the evolution for the first eigenvalue of $p$-Laplacian along the Yamabe flow and also in [3] shown the monotonicity of eigenvalues of Witten-Laplace operator along the Ricci-Bourguignon flow. Also for more details in a case of $p$-Laplacian operator, Wang in [17], has shown the eigenvalue estimate for the weighted $p$-Laplacian and later in [18] shown the gradient estimate on the weighted $p$-Laplace heat equation. Beside what mentioned before A. Abolarinwa in [1], has studied the evolution and monotonicity of the first eigenvalue of $p$-Laplacian under the Ricci-harmonic flow and also you can find some useful results in eigenvalue monotonicity of the $p$-Laplace operator under the Ricci flow in [20], also Cao and Songbo Hou have worked on monotonicity of the first eigenvalue under Ricci flow and you can see their results in $[8,12]$. Finally we will use some results of $[19]$ in this work.

In this paper we will investigate the evolution of the first eigenvalue of $p$-Laplacian operator and then we will find some bounds in a case of Bianchi classes.

\section{Preliminaries and evolution equation}

Let $(M, g)$ be a locally homogeneous closed 3-manifold, there are nine classes of such manifolds. They are divided into two groups, the first consists of $H(3)$, $H(2) \times \mathbb{R}^{1}$ and $S o(3) \times \mathbb{R}^{1}$ and the other one includes $\mathbb{R}^{3}, S U(2), S L(2, \mathbb{R})$, Heisenberg, $E(1,1)$ and $E(2)$ which are called Bianchi classes. Milnor [15], has provided a frame $\left\{X_{i}\right\}_{i=1}^{3}$ where both the metric and Ricci tensors are diagonalized and this property is preserved by the Ricci flow (1). Now let $\{\theta\}_{i=1}^{3}$ be a dual to Milnor's frame, we 
consider the metric $g(t)$ as

$$
g(t)=A(t)\left(\theta_{1}\right)^{2}+B(t)\left(\theta_{2}\right)^{2}+C(t)\left(\theta_{3}\right)^{2},
$$

then the Ricci flow becomes a system of ODE with three variables $\{A(t), B(t), C(t)\}$. Consider $M$ as a compact Riemannian manifold and $u: M \longrightarrow \mathbb{R}$ be a smooth function on $M$ or we can consider $u \in W^{1, p}(M)$ the Sobolev space. The $p$-Laplacian of $u$ for $1<p<\infty$ is defined as

$$
\begin{aligned}
\Delta_{p} u & =\operatorname{div}\left(|\nabla u|^{p-2} \nabla u\right) \\
& =|\nabla u|^{p-2} \Delta u+(p-2)|\nabla u|^{p-4}(\text { Hess } u)(\nabla u, \nabla u),
\end{aligned}
$$

where

$$
\left(\text { Hessu) }(X, Y)=\nabla(\nabla u)(X, Y)=X .(Y . u)-\left(\nabla_{X} Y\right) . u \quad X, Y \in \chi(M) .\right.
$$

In this case we say that $\lambda$ is an eigenvalue of $p$-Laplace operator whenever there exist a function $u$ on $M$ such that

$$
\begin{gathered}
\Delta_{p} u=-\lambda|u|^{p-1} u \\
\lambda(t)=\inf \left\{\int_{M}|\nabla u|^{p} d \mu \mid u\right. \\
\left.\in W^{1, p}(M) \backslash\{0\}, \int_{M}|u|^{p} d \mu=1\right\} .
\end{gathered}
$$

The theorem below from [19] gives us the continuity of the first eigenvalue of $p$ Laplace operator.

Theorem 2.1. If $g_{1}$ and $g_{2}$ are two metrics which satisfy

$$
(1+\varepsilon)^{-1} g_{1} \leq g_{2} \leq(1+\varepsilon) g_{1},
$$

then for any $p>1$, we have

$$
(1+\varepsilon)^{-\left(n+\frac{p}{2}\right)} \lambda\left(g_{2}\right) \leq \lambda\left(g_{1}\right) \leq(1+\varepsilon)^{\left(n+\frac{p}{2}\right)} \lambda\left(g_{2}\right) .
$$

In particular $\lambda(g(t))$ is a continuous function in a t-variable.

Discussing about the monotonicity of the first eigenvalue of the $p$-Laplace operator powerfully is dependent to the differentiability of the eigenvalue function. In this section we are following the X. Cao's argument [4], where we introduce the smooth eigenvalue function $\lambda(u, t)$ which is smooth then we can write the monotonicity of $\lambda(t)$. We assume at time $t_{0}, u_{0}=u\left(t_{0}\right)$ is eigenfunction for the first eigenvalue $\lambda\left(t_{0}\right)$ of $p$-Laplacian. Then we have

$$
\int_{M}\left|u\left(t_{0}\right)\right|^{p} d \mu_{g\left(t_{0}\right)}=1 .
$$

We consider the following smooth function

$$
\varphi(t):=u_{0}\left[\frac{\operatorname{det}\left(g_{i j}\left(t_{0}\right)\right)}{\operatorname{det}\left(g_{i j}(t)\right)}\right]^{\frac{1}{2(p-1)}},
$$

and normalize this smooth function as

$$
u(t)=\frac{\varphi(t)}{\left(\int_{M}|\varphi(t)|^{p} d \mu\right)^{\frac{1}{p}}},
$$


under the Ricci flow. Now we define a general smooth function as

where $u$ is any smooth function satisfying

$$
\begin{aligned}
\lambda(u, t) & :=-\int_{M} \Delta_{p} u(t) \cdot u(t) d \mu \\
& =\int_{M}|\nabla u|^{p} d \mu,
\end{aligned}
$$

$$
\int_{M}|u|^{p} d \mu=1 \quad \text { and } \quad \int_{M}|u|^{p-2} u d \mu=0 .
$$

In general $\lambda(u, t)$ is not equal to $\lambda(t)$. But at time $t_{0}$ we conclude that

$$
\lambda\left(u\left(t_{0}\right), t_{0}\right)=\lambda\left(t_{0}\right) .
$$

Now we are ready to give an evolution formula for $\left.\lambda(u, t)\right|_{t=t_{0}}$ along the normalized Ricci flow in a case of 3 -homogeneous manifold. In this case it is not hard to see $R=r$ where $R$ is scalar curvature and $r$ is as same as what explained in the definition of the normalized Ricci flow (2).

Proposition 2.2. Let $(M, g(t))$ be a solution of the normalized Ricci flow (2), on a locally homogeneous 3-manifold. If $\lambda_{1, p}(t)$ denotes the first eigenvalue of the p-Laplacian (3), then

$$
\left.\frac{d}{d t} \lambda(u, t)\right|_{t=t_{0}}=p \int|\nabla u|^{p-2} R_{i j} u^{i} u^{j} d \mu-\frac{p R}{3} \lambda\left(t_{0}\right),
$$

where $u^{i}=\nabla^{i} u$ and $u^{j}=\nabla^{j} u$.

Proof. By the direct computation it will be easy to see that under the normalized Ricci flow (2) we have

$$
\frac{\partial}{\partial t}|\nabla u|^{p}=p|\nabla u|^{p-2}\left(\left(R^{i j}-\frac{r}{3} g^{i j}\right) u_{i} u_{j}+u_{i} \frac{\partial u_{i}}{\partial t}\right),
$$

and also

$$
\frac{\partial}{\partial t}(d \mu)=-(R-r) d \mu
$$

The function $\lambda(u, t)$ is smooth so it concludes that

$$
\begin{aligned}
\left.\frac{d}{d t} \lambda(u, t)\right|_{t=t_{0}} & =\int_{M} \frac{\partial}{\partial t}|\nabla u|^{p} d \mu-\int_{M}|\nabla u|^{p}(R-r) d \mu \\
& =p \int_{M}|\nabla u|^{p-2}\left(\left(R^{i j}-\frac{r}{3} g^{i j}\right) u_{i} u_{j}\right) d \mu+p \int_{M}|\nabla u|^{p-2} u_{i} \frac{\partial u_{i}}{\partial t} d \mu \\
& -\int_{M}|\nabla u|^{p}(R-r) d \mu .
\end{aligned}
$$

As we mentioned before in homogeneous manifold $R=r$ also the condition $\int_{M}|u|^{p} d \mu=$ 1 results that

$$
\int_{M}|\nabla u|^{p-2} u_{i} \frac{\partial u_{i}}{\partial t} d \mu=0
$$


Hence

$$
\begin{aligned}
\left.\frac{d}{d t} \lambda(u, t)\right|_{t=t_{0}} & =p \int_{M}|\nabla u|^{p-2} R^{i j} u_{i} u_{j} d \mu-\frac{p R}{3} \lambda\left(t_{0}\right) \\
& =p \int_{M}|\nabla u|^{p-2} R_{i j} u^{i} u^{j} d \mu-\frac{p R}{3} \lambda\left(t_{0}\right),
\end{aligned}
$$

which implies what we looking for.

\section{Estimate of $\lambda(t)$ ON Bianchi Classes}

In this section we are going to give some useful bounds for $\lambda(t)$ separately in Bianchi classes. Before Hou in [11] has given bounds for the first eigenvalue of $\Delta$ in a case of $u>0$ under the backward Ricci flow and also he proved the eigenvalue evolves toward zero in a case that the backward Ricci flow converges to a sub-Riemannian geometry by a proper rescaling. Later Razavi and Korouki in [13] have done similar work for the first eigenvalue of $(-\Delta-R)$ under the Ricci flow.

Remark 3.1. In homogeneous condition and in a case of the un-normalized Ricci flow we get that

$$
\left.\frac{d}{d t} \lambda(u, t)\right|_{t=t_{0}}=p \int_{M}|\nabla u|^{p-2} R_{i j} u_{i} u_{j} d \mu
$$

Now we study the behavior of the first eigenvalue of $p$-Laplacian in each classes separately.

\section{Case 1: $\mathbb{R}^{3}$}

In this case all metrics are flat, so for all $t \geq 0$ we have $g(t)=g_{0}$ where $g_{0}$ is initial metric, therefore $\lambda(t)$ is constant.

\section{Case 2: Heisenberg}

This class is isomorphic to the set of upper-triangular $3 \times 3$ matrices endowed with the usual matrix multiplication. Under the metric $g_{0}$ we choose a frame $\left\{X_{i}\right\}_{i=1}^{3}$ in which

$$
\left[X_{2}, X_{3}\right]=X_{1}, \quad\left[X_{3}, X_{1}\right]=0, \quad\left[X_{1}, X_{2}\right]=0,
$$

also under the normalization $A_{0} B_{0} C_{0}=1$ we have

$$
\begin{aligned}
& R_{11}=\frac{1}{2} A^{3}, \quad R_{22}=-\frac{1}{2} A^{2} B, \quad R_{33}=-\frac{1}{2} A^{2} C, \\
& R=-\frac{1}{2} A^{2} .
\end{aligned}
$$

Theorem 3.2. Let $\lambda(t)$ be the first eigenvalue of p-Laplace operator on Heisenberg Riemannian manifold $\left(\mathcal{H}^{3}, g_{0}\right)$ and also assume that $B_{0} \geq C_{0}$. Then in a sufficient neighborhood as $[0, t]$, the quantities $\lambda(t) e^{\int_{0}^{t}\left(-\frac{2}{3} p A^{2}\right) d \tau}$ is nondecreasing and $\lambda(t) e^{\int_{0}^{t}\left(\frac{1}{3} p A^{2}\right) d \tau}$ is nonincreasing along the normalized Ricci flow (2), where

$$
-\frac{1}{3} p A^{2} \lambda\left(t_{0}\right) \leq\left.\frac{d}{d t} \lambda(u, t)\right|_{t=t_{0}} \leq \frac{2}{3} p A^{2} \lambda\left(t_{0}\right)
$$


Proof. Under the proposition 2.2 and Ricci coordinates in Hiesenberg case, we get

$$
\begin{aligned}
\left.\frac{d}{d t} \lambda(u, t)\right|_{t=t_{0}} & =\int_{\mathcal{H}^{3}}|\nabla u|^{p-2}\left[g_{11} \nabla^{1} u \nabla^{1} u-g_{22} \nabla^{2} u \nabla^{2} u\right. \\
& \left.-g_{33} \nabla^{3} u \nabla^{3} u\right] d \mu-\frac{p}{3} R \lambda\left(t_{0}\right) \\
& \leq \frac{1}{2} p A^{2} \int_{\mathcal{H}^{3}}|\nabla u|^{p-2}\left[g_{11} \nabla^{1} u \nabla^{1} u+g_{22} \nabla^{2} u \nabla^{2} u\right. \\
& \left.+g_{33} \nabla^{3} u \nabla^{3} u\right] d \mu-\frac{p}{3} R \lambda\left(t_{0}\right) \\
& =\frac{1}{2} p A^{2} \lambda\left(t_{0}\right)-\frac{p}{3} R \lambda\left(t_{0}\right) .
\end{aligned}
$$

By substituting $R$ into formula (7) we obtain

$$
\left.\frac{d}{d t} \lambda(u, t)\right|_{t=t_{0}} \leq \frac{2}{3} p A^{2} \lambda\left(t_{0}\right)
$$

Since $\lambda(f, t)$ is smooth function with respect to time $t$, hence in any sufficiently small neighborhood of $t_{0}$, we have

$$
\frac{d}{d t} \lambda(f(t), t) \leq \frac{2}{3} p A^{2} \lambda(f(t), t)
$$

Since $t_{0}$ is arbitrary then for any $t \in[0, T)$ the inequality (8) holds and it implies

$$
\frac{d}{d t}\left(\lambda(t) e^{\int_{0}^{t}\left(-\frac{2}{3} p A^{2}\right) d \tau}\right) \geq 0
$$

Therefore the quantity $\lambda(t) e^{\int_{0}^{t}\left(-\frac{2}{3} p A^{2}\right) d \tau}$ is nondecreasing. Also in a similar way we have

$$
\begin{aligned}
\left.\frac{d}{d t} \lambda(u, t)\right|_{t=t_{0}} & \geq \frac{1}{2} p A^{2} \int_{\mathcal{H}^{3}}|\nabla u|^{p-2}\left[-g_{11} \nabla^{1} u \nabla^{1} u-g_{22} \nabla^{2} u \nabla^{2} u\right. \\
& \left.-g_{33} \nabla^{3} u \nabla^{3} u\right] d \mu-\frac{p}{3} R \lambda\left(t_{0}\right) \\
& \geq-\frac{1}{2} p A^{2} \lambda\left(t_{0}\right)-\frac{p}{3} R \lambda\left(t_{0}\right) \\
& =-\frac{1}{3} p A^{2} \lambda\left(t_{0}\right) .
\end{aligned}
$$

Which implies what we are looking for.

Remark 3.3. In this case by [11], for the tensors $A, B$ and $C$ we have

$$
\begin{aligned}
& A=A_{0}\left(1+\frac{16}{3} R_{0} t\right)^{-\frac{1}{2}}, \quad B=B_{0}\left(1+\frac{16}{3} R_{0} t\right)^{\frac{1}{4}}, \\
& C=C_{0}\left(1+\frac{16}{3} R_{0} t\right)^{\frac{1}{4}},
\end{aligned}
$$


where $R_{0}=-\frac{1}{2} A_{0}^{2}$. Now by substituting these formulas into the formula (7) which is hold for arbitrary $t_{0}$, and integrating from both sides in $\left[t_{0}, t\right]$, we get

$$
\ln \lambda(t) \leq \frac{1}{2} \cdot \frac{p A_{0}^{2}}{1+\frac{16}{3} R_{0}} \ln \left(1+\frac{16}{3} R_{0} t\right),
$$

and similarly

$$
\ln \lambda(t) \geq-\frac{1}{2} \cdot \frac{p A_{0}^{2}}{1+\frac{16}{3} R_{0}} \ln \left(1+\frac{16}{3} R_{0} t\right) .
$$

\section{Case 3: $\mathrm{E}(2)$}

Manifold E(2) is the group of isometries of Euclidian plane. In this case we have an Einstein metric and Ricci flow converges exponentially to flat metrics. Dependent to the metric $g_{0}$ we choose the frame $\left\{X_{i}\right\}_{i=0}^{3}$ such that

$$
\left[X_{2}, X_{3}\right]=X_{1}, \quad\left[X_{3}, X_{1}\right]=X_{2}, \quad\left[X_{1}, X_{2}\right]=0,
$$

In this case under the normalization $A_{0} B_{0} C_{0}=1$ we have

$$
\begin{aligned}
& R_{11}=\frac{1}{2} A\left(A^{2}-B^{2}\right), \quad R_{22}=\frac{1}{2} B\left(B^{2}-A^{2}\right), \\
& R_{33}=-\frac{1}{2} C(A-B)^{2}, \quad R=-\frac{1}{2}(A-B)^{2} .
\end{aligned}
$$

X. Cao in [7] has proved that for initial tensors $A_{0}$ and $B_{0}$

- If $A_{0}=B_{0}$ then $A=B$, in this case $g(t)=g_{0}$ where $g_{0}$ is constant.

- If $A_{0}>B_{0}$ then $A>B$ in this case we have

Theorem 3.4. Consider $\lambda(t)$ as a first eigenvalue of $p$-Laplace operator on 3-homogeneous Riemannian manifold $\left(E(2), g_{0}\right)$ and also let $A_{0}>B_{0}$ then in a sufficient neighborhood as $[0, t]$, the quantities $\lambda(t) e^{\int_{0}^{t}-\frac{1}{2} p\left(\left(A^{2}-B^{2}\right)-\frac{1}{3}(A-B)^{2}\right) d \tau}$ and $\lambda(t) e^{\int_{0}^{t} \frac{1}{2} p\left(\left(A^{2}-B^{2}\right)-\frac{1}{3}(A-B)^{2}\right) d \tau}$ are non-decreasing and non-increasing along the normalized Ricci flow (2), respectively, where

$$
\begin{aligned}
-\frac{1}{2} p\left(\left(A^{2}-B^{2}\right)-\frac{1}{3}(A-B)^{2}\right) \lambda\left(t_{0}\right) & \leq\left.\frac{d}{d t} \lambda(u, t)\right|_{t=t_{0}} \\
& \leq \frac{1}{2} p\left(\left(A^{2}-B^{2}\right)-\frac{1}{3}(A-B)^{2}\right) \lambda\left(t_{0}\right) .
\end{aligned}
$$

Proof. Since by [6] we have $A>B$ and also under the proposition 2.2, we get

$$
\begin{gathered}
\left.\frac{d}{d t} \lambda(u, t)\right|_{t=t_{0}}=p \int_{E(2)}|\nabla u|^{p-2}\left[\frac{1}{2} A\left(A^{2}-B^{2}\right) \nabla^{1} u \nabla^{1} u-\frac{1}{2} B\left(A^{2}-B^{2}\right) \nabla^{2} u \nabla^{2} u\right. \\
\left.-\frac{1}{2} C(A-B)^{2} \nabla^{3} u \nabla^{3} u\right] d \mu-\frac{p}{3} R,
\end{gathered}
$$


where by the assumption $A_{0}>B_{0}$ we have

$$
\begin{aligned}
\left.\frac{d}{d t} \lambda(u, t)\right|_{t=t_{0}} & \geq p \int_{E(2)}|\nabla u|^{p-2}\left[-\frac{1}{2} A\left(A^{2}-B^{2}\right) \nabla^{1} u \nabla^{1} u-\frac{1}{2} B\left(A^{2}-B^{2}\right) \nabla^{2} u \nabla^{2} u\right. \\
& \left.-\frac{1}{2} C\left(A^{2}-B^{2}\right) \nabla^{3} u \nabla^{3} u\right] d \mu-\frac{p}{3} R \lambda\left(t_{0}\right) \\
& \geq-\frac{1}{2} p\left(A^{2}-B^{2}\right) \lambda\left(t_{0}\right)-\frac{p}{3} R \lambda\left(t_{0}\right) .
\end{aligned}
$$

In a similar way

$$
\left.\frac{d}{d t} \lambda(u, t)\right|_{t=t_{0}} \leq \frac{1}{2} p\left(A^{2}-B^{2}\right) \lambda\left(t_{0}\right)-\frac{p}{3} R \lambda\left(t_{0}\right),
$$

now apply $R$ from above, since $t_{0}$ is arbitrary it implies what mentioned before in the theorem.

\section{Case 4: $\mathrm{E}(1,1)$}

Manifold $\mathrm{E}(1,1)$ is the group of isometries of the plane with flat Lorentz metric, there is no Einstein metric here and Ricci flow fails to converge, they all are asymptotically cigar degeneracies. For a given metric $g_{0}$ similarly by a frame $\left\{X_{i}\right\}_{i=0}^{3}$ we have

$$
\left[X_{1}, X_{2}\right]=0, \quad\left[X_{2}, X_{3}\right]=-X_{1}, \quad\left[X_{3}, X_{1}\right]=X_{2} .
$$

Also under the normalization $A_{0} B_{0} C_{0}=1$ we obtain

$$
\begin{array}{ll}
R_{11}=\frac{1}{2} A\left(A^{2}-C^{2}\right), & R_{22}=-\frac{1}{2} B(A+C)^{2}, \\
R_{33}=\frac{1}{2} C\left(C^{2}-A^{2}\right), & R=-\frac{1}{2}(A+C)^{2} .
\end{array}
$$

Theorem 3.5. Let $\lambda(t)$ denotes the first eigenvalue of $p$-Laplace operator on 3-homogeneous Riemannian manifold $\left(E(1,1), g_{0}\right)$ then in a sufficient neighborhood $[0, t]$ we get

- If $A_{0}=C_{0}$ then $\lambda(t) e^{\int_{0}^{t}\left(-\frac{1}{3} p(A+C)^{2}\right) d \tau}$ and $\lambda(t) e^{\int_{0}^{t}\left(\frac{1}{3} p(A+C)^{2}\right) d \tau}$ are nondecreasing and non-increasing along the normalized Ricci flow (2), respectively, where

$$
\left(-\frac{1}{3} p(A+C)^{2}\right) \lambda\left(t_{0}\right) \leq\left.\frac{d}{d t} \lambda(u, t)\right|_{t=t_{0}} \leq\left(\frac{1}{3} p(A+C)^{2}\right) \lambda\left(t_{0}\right) .
$$

- If $A_{0}>C_{0}$ then $\lambda(t) e^{\int_{0}^{t}-\frac{1}{2} p\left(\left(A^{2}-C^{2}\right)-\frac{1}{3}(A+C)^{2}\right) d t}$ and $\lambda(t) e^{\int_{0}^{t}\left(\frac{2}{3} p(A+C)^{2}\right) d t}$ are non-decreasing and non-increasing respectively, where

$$
-\frac{1}{2} p\left(\left(A^{2}-C^{2}\right)-\frac{1}{3}(A+C)^{2}\right) \lambda\left(t_{0}\right) \leq\left.\frac{d}{d t} \lambda(u, t)\right|_{t=t_{0}} \leq\left(\frac{2}{3} p(A+C)^{2}\right) \lambda\left(t_{0}\right) .
$$

Proof. In the case of $A_{0}=C_{0}$ by [6] we get that $A=C$, it is easy to see

$$
R_{11}=R_{33}=0
$$


which means

$$
\left.\frac{d}{d t} \lambda(u, t)\right|_{t=t_{0}} \leq \frac{1}{2} p(A+C)^{2} \lambda\left(t_{0}\right)-\frac{p}{3} R \lambda\left(t_{0}\right) .
$$

Similarly in a case of $A_{0}>C_{0}$ it is easy to get

$$
\begin{aligned}
\left.\frac{d}{d t} \lambda(u, t)\right|_{t=t_{0}} \geq p \int_{E(1,1)}|\nabla u|^{p-2}[ & -\frac{1}{2} A\left(A^{2}-C^{2}\right) \nabla^{1} u \nabla^{1} u-\frac{1}{2} B\left(A^{2}-C^{2}\right) \nabla^{2} u \nabla^{2} u \\
& \left.-\frac{1}{2} C\left(A^{2}-C^{2}\right) \nabla^{3} u \nabla^{3} u\right] d \mu-\frac{p}{3} R \lambda\left(t_{0}\right),
\end{aligned}
$$

and in a similar way

$$
\left.\frac{d}{d t} \lambda(u, t)\right|_{t=t_{0}} \leq \frac{1}{2} p(A+C)^{2} \lambda\left(t_{0}\right)-\frac{p}{3} R \lambda\left(t_{0}\right),
$$

now similar to the above theorems, the proof is completed.

\section{Case 5: SU(2)}

Similarly in this class we have Einstein metrics and Ricci flow converges exponentially in to these metrics, also by the frame $\left\{X_{i}\right\}_{i=0}^{3}$ we have

$$
\left[X_{2}, X_{3}\right]=X_{1}, \quad\left[X_{3}, X_{1}\right]=X_{2}, \quad\left[X_{1}, X_{2}\right]=X_{3} .
$$

In this case under the normalization $A_{0} B_{0} C_{0}=1$, we have

where

$$
\begin{aligned}
R_{11} & =\frac{1}{2} A\left[A^{2}-(B-C)^{2}\right], \quad R_{22}=\frac{1}{2} B\left[B^{2}-(A-C)^{2}\right], \\
R_{33} & =\frac{1}{2} C\left[C^{2}-(A-B)^{2}\right],
\end{aligned}
$$

$$
R=\frac{1}{2}\left[A^{2}-(B-C)^{2}\right]+\frac{1}{2}\left[B^{2}-(A-C)^{2}\right]+\frac{1}{2}\left[C^{2}-(A-B)^{2}\right] .
$$

Theorem 3.6. Consider $\lambda(t)$ as a first eigenvalue of $p$-Laplace operator on 3homogeneous Riemannian manifold $\left(S U(2), g_{0}\right)$ then there is a time $\tilde{t}$ and the interval $[\tilde{t}, t]$ in which we have

- If $A_{0}=B_{0}=C_{0}$ then $\lambda(t)=\lambda(0)$.

- If $A_{0}=B_{0}>C_{0}$ then $\lambda(t) e^{\int_{\tilde{t}}^{t}\left(p(B-C)^{2}-\frac{1}{2} p\left(A^{2}+C^{2}\right)\right) d \tau} \quad$ and $\quad \lambda(t) e^{\int_{\tilde{t}}^{t}\left(\frac{1}{2} p A^{2}\left((B-C)^{2}+1\right)\right) d \tau}$ are non-decreasing and non-increasing respectively, where

$$
\left(p(B-C)^{2}-\frac{1}{2} p\left(A^{2}+C^{2}\right)\right) \lambda\left(t_{0}\right) \leq\left.\frac{d}{d t} \lambda(u, t)\right|_{t=t_{0}} \leq\left(\frac{1}{2} p A^{2}\left((B-C)^{2}+1\right)\right) \lambda\left(t_{0}\right) .
$$

- If $A_{0}>B_{0} \geq C_{0}$ then

$\lambda(t) e^{\int_{\tilde{t}}^{t} \frac{1}{2} p\left(\left(C^{2}-(A-C)^{2}\right)-\left(A^{2}-(B-C)^{2}\right)\right) d \tau} \quad$ and $\quad \lambda(t) e^{\int_{\tilde{t}}^{t} \frac{1}{2} p\left(\left(A^{2}-(B-C)^{2}\right)-\left(C^{2}-(A-C)^{2}\right)\right) d \tau}$ are non-decreasing and non-increasing along the normalized Ricci flow (2) respectively, where

$$
\left.\frac{d}{d t} \lambda(u, t)\right|_{t=t_{0}} \geq \frac{1}{2} p\left(\left(C^{2}-(A-C)^{2}\right)-\left(A^{2}-(B-C)^{2}\right)\right) \lambda\left(t_{0}\right),
$$




$$
\begin{aligned}
& \text { and } \\
& \left.\frac{d}{d t} \lambda(u, t)\right|_{t=t_{0}} \leq \frac{1}{2} p\left(\left(A^{2}-(B-C)^{2}\right)-\left(C^{2}-(A-C)^{2}\right)\right) \lambda\left(t_{0}\right) .
\end{aligned}
$$

Proof. By X. Cao [7], the proof of first and second section will be easy and similarly for the third section we have

$$
\begin{aligned}
\left.\frac{d}{d t} \lambda(u, t)\right|_{t=t_{0}} & \leq p \int_{S U(2)}|\nabla u|^{p-2}\left[\frac{1}{2} A\left(A^{2}-(B-C)^{2}\right) \nabla^{1} u \nabla^{1} u+\frac{1}{2} B\left(A^{2}-(A-C)^{2}\right) \nabla^{2} u \nabla^{2} u\right. \\
& \left.+\frac{1}{2} C\left(A^{2}-(A-B)^{2}\right) \nabla^{3} u \nabla^{3} u\right] d \mu-\frac{p}{3} R \lambda\left(t_{0}\right) \\
& \leq p \int_{S U(2)}|\nabla u|^{p-2}\left[\frac{1}{2} A\left(A^{2}-(B-C)^{2}\right) \nabla^{1} u \nabla^{1} u+B\left(A^{2}-(B-C)^{2}\right) \nabla^{2} u \nabla^{2} u\right. \\
& \left.+C\left(A^{2}-(B-C)^{2}\right) \nabla^{3} u \nabla^{3} u\right] d \mu-\frac{p}{3} R \lambda\left(t_{0}\right) \\
& \leq \frac{1}{2} p\left(A^{2}-(B-C)^{2}\right) \lambda\left(t_{0}\right)-\frac{p}{3} R \lambda\left(t_{0}\right) .
\end{aligned}
$$

Also in a similar way

$$
\begin{aligned}
\left.\frac{d}{d t} \lambda(u, t)\right|_{t=t_{0}} & \geq p \int_{S U(2)}|\nabla u|^{p-2}\left[\frac{1}{2} A\left(C^{2}-(B-C)^{2}\right) \nabla^{1} u \nabla^{1} u+\frac{1}{2} B\left(C^{2}-(A-C)^{2}\right) \nabla^{2} u \nabla^{2} u\right. \\
& \left.+\frac{1}{2} C\left(C^{2}-(A-B)^{2}\right) \nabla^{3} u \nabla^{3} u\right] d \mu-\frac{p}{3} R \lambda\left(t_{0}\right) \\
& \geq p \int_{S U(2)}|\nabla u|^{p-2}\left[\frac{1}{2} A\left(C^{2}-(A-C)^{2}\right) \nabla^{1} u \nabla^{1} u+\frac{1}{2} B\left(C^{2}-(A-C)^{2}\right) \nabla^{2} u \nabla^{2} u\right. \\
& \left.+\frac{1}{2} C\left(C^{2}-(A-C)^{2}\right) \nabla^{3} u \nabla^{3} u\right] d \mu-\frac{p}{3} R \lambda\left(t_{0}\right) \\
& \geq \frac{1}{2} p\left(C^{2}-(A-C)^{2}\right) \lambda\left(t_{0}\right)-\frac{p}{3} R \lambda\left(t_{0}\right),
\end{aligned}
$$

which if you substitute $R$, it is completed the proof.

\section{Case 6: $\mathrm{SL}(2, \mathbb{R})$}

On $S L(2, \mathbb{R})$ there is no Einstein metric and the Ricci flow doesn't converge and develops a pancake degeneracy, also by the frame $\left\{X_{i}\right\}_{i=0}^{3}$, we get

$$
\left[X_{2}, X_{3}\right]=-X_{1}, \quad\left[X_{3}, X_{1}\right]=X_{2}, \quad\left[X_{1}, X_{2}\right]=X_{3},
$$

in this case we also have

$$
\begin{aligned}
& R_{11}=\frac{1}{2} A\left[A^{2}-(B-C)^{2}\right], \quad R_{22}=\frac{1}{2} B\left[B^{2}-(A+C)^{2}\right], \\
& R_{33}=\frac{1}{2} C\left[C^{2}-(A+B)^{2}\right] .
\end{aligned}
$$

In which

$$
R=\frac{1}{2}\left[A^{2}-(B-C)^{2}\right]+\frac{1}{2}\left[B^{2}-(A+C)^{2}\right]+\frac{1}{2}\left[C^{2}-(A+B)^{2}\right] .
$$


Theorem 3.7. Let $\lambda(t)$ be the first eigenvalue of p-Laplace operator on 3-homogeneous Riemannian manifold $\left(S L(2, \mathbb{R}), g_{0}\right)$ and also let there is a time $\tilde{t}$ and interval $[\tilde{t}, t]$ we get

- If $A>B=C$ then

$\lambda(t) e^{\int_{\hat{t}}^{t} \frac{1}{2} p\left(\left(C^{2}-(A+B)^{2}\right)-A^{2}\right) d \tau} \quad$ and $\quad \lambda(t) e^{\int_{\hat{t}}^{t} \frac{1}{2} p\left(A^{2}-\left(C^{2}-(A+B)^{2}\right)\right) d \tau}$,

are non-decreasing and non-increasing along the normalized Ricci flow (2), where

$$
\left.\frac{d}{d t} \lambda(u, t)\right|_{t=t_{0}} \geq \frac{1}{2} p\left(\left(C^{2}-(A+B)^{2}\right)-A^{2}\right) \lambda\left(t_{0}\right),
$$

and

$$
\left.\frac{d}{d t} \lambda(u, t)\right|_{t=t_{0}} \leq \frac{1}{2} p\left(A^{2}-\left(C^{2}-(A+B)^{2}\right)\right) \lambda\left(t_{0}\right) .
$$

- If $A \leq B-C$ then

$$
\lambda(t) e^{\int_{\hat{t}}^{t}-\frac{1}{2} p\left((B-C)^{2}-A^{2}+B^{2}\right) d \tau} \quad \text { and } \quad \lambda(t) e^{\int_{\hat{t}}^{t}-\frac{1}{2} p C^{2} d \tau},
$$

are non-decreasing and non-increasing along the normalized Ricci flow (2), where

$$
-\frac{1}{2} p\left((B-C)^{2}-A^{2}+B^{2}\right) \lambda\left(t_{0}\right) \leq\left.\frac{d}{d t} \lambda(u, t)\right|_{t=t_{0}} \leq-\frac{1}{2} p C^{2} .
$$

Proof. By X. Cao $[6,7]$ we can easily calculate that

- for the first section we have

$$
\begin{aligned}
\left.\frac{d}{d t} \lambda(u, t)\right|_{t=t_{0}} & \geq p \int_{S L(2, \mathbb{R})}|\nabla u|^{p-2}\left[\frac{1}{2} A\left(C^{2}-(A+C)^{2}\right) \nabla^{1} u \nabla^{1} u+\frac{1}{2} B\left(C^{2}-(A+C)^{2}\right) \nabla^{2} u \nabla^{2} u\right. \\
& \left.+\frac{1}{2} C\left(C^{2}-(A+C)^{2}\right) \nabla^{3} u \nabla^{3} u\right] d \mu-\frac{p}{3} r \lambda\left(t_{0}\right) \\
& \geq \frac{1}{2} p\left(C^{2}-(A+C)^{2}\right) \lambda\left(t_{0}\right)-\frac{p}{3} r \lambda\left(t_{0}\right),
\end{aligned}
$$

also similarly we get

$$
\left.\frac{d}{d t} \lambda(u, t)\right|_{t=t_{0}} \leq \frac{1}{2} p\left(A^{2}-(B-C)^{2}\right) \lambda\left(t_{0}\right)-\frac{p}{3} R \lambda\left(t_{0}\right) .
$$

- For the second section also we get

$$
\begin{aligned}
\left.\frac{d}{d t} \lambda(u, t)\right|_{t=t_{0}} & =p \int_{S L(2, \mathbb{R})}|\nabla u|^{p-2}\left[-\frac{1}{2} A\left((B-C)^{2}-A^{2}\right) \nabla^{1} u \nabla^{1} u+\frac{1}{2} B\left(B^{2}-(A+c)^{2}\right) \nabla^{2} u \nabla^{2} u\right. \\
& \left.-\frac{1}{2} C\left((A+B)^{2}-C^{2}\right) \nabla^{3} u \nabla^{3} u\right] d \mu-\frac{p}{3} R \lambda\left(t_{0}\right) \\
& \leq \frac{1}{2} p B^{2} \lambda\left(t_{0}\right),
\end{aligned}
$$

also under consideration $A \leq B-C$ we have

$$
(A+B)^{2}>(B-C)^{2},
$$


now we can get

$$
\begin{aligned}
\left.\frac{d}{d t} \lambda(u, t)\right|_{t=t_{0}} & \geq p \int_{S L(2, \mathbb{R})}|\nabla u|^{p-2}\left[-\frac{1}{2} A\left((B-C)^{2}-A^{2}\right) \nabla^{1} u \nabla^{1} u-\frac{1}{2} B\left((B-C)^{2}-A^{2}\right) \nabla^{2} u \nabla^{2} u\right. \\
& \left.-\frac{1}{2} C\left((B-C)^{2}-A^{2}\right) \nabla^{3} u \nabla^{3} u\right] d \mu-\frac{p}{3} R \lambda\left(t_{0}\right) \\
& \geq-\frac{1}{2} p\left((B-C)^{2}-A^{2}\right) \lambda\left(t_{0}\right)-\frac{p}{3} R \lambda\left(t_{0}\right),
\end{aligned}
$$

now we should substitute $R$, this is making the proof complete.

\section{REFERENCES}

[1] A. Abolarinwa, Evolution and monotonicity of the first eigenvalue of p-Laplacian under the Ricci harmonic flow, J. Appl. Anal., 21(2) (2015), 147-160.

[2] S. Azami, Eigenvalue variation of the p-Laplacian under the Yamabe flow, Cogent mathematics, 3 (2016), 1236566.

[3] S. Azami, Monotonicity of eigenvalues of Witten-Laplace operator along the RicciBourguignon flow, AIMS mathematics, 2(2)(2017), 230-243.

[4] X. Cao, Eigenvalues of $\left(-\Delta+\frac{R}{2}\right)$ on manifolds with nonnegative curvature operator, Math. Ann., 337(2) (2007), 435-442.

[5] X. Cao, First eigenvalues of geometric operators under the Ricci flow, Proc. Amer. Math. Soc, 136(11) (2008), 4075-4078.

[6] X. Cao, J. Guckenheimer and L. Saloff-Coste, The backward behavior of the Ricci and cross curvature flows on $S L(2, \mathbb{R})$, Comm. Anal. Geom., 17(4) (2009), 777-796.

[7] X. Cao and L. Saloff-Coste, Backward Ricci flow on locally homogeneous three-manifolds, Comm. Anal. Geom., 12(2), (2009) 305-325.

[8] X. Cao, S. Hou and J. Ling, Estimates and monotonicity of the first eigenvalue under the Ricci flow, Math. Ann. 345 (2012), no. 2, 451-463.

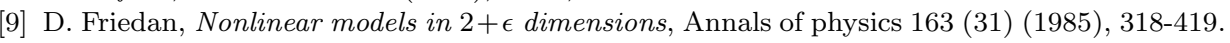

[10] R. Hamilton, Three-manifolds with positive Ricci curvature, J. Diff. Geom. 17 (1982), 255306.

[11] S. Hou, Eigenvalues under the backward Ricci flow on locally homogeneous closed 3manifolds, Acta Mathematica Sinica, English series, 136(11) (2018), 1179-1194.

[12] S. Hou, Eigenvalues under the Ricci flow of model geometries, (Chinese) Acta Math. Sinica (Chin. ser.) 60 (2017), no. 4, 583-594.

[13] F. Korouki and A. Razavi, Bounds for the first eigenvalue of $(-\Delta-R)$ under the Ricci flow on Bianchi classes, Bull. Braz. Math. Soc, (2019).

[14] J. Li, Eigenvalues and energy functionals with monotonicity formula under Ricci flow, Math. Ann., 338 (2007), 927-946.

[15] J. Milnor, Curvatures of left invariant metrics on Lie groups, Adv. Math., 21(3) (1976), 293-329.

[16] G. Perelman, The entropy formula for the Ricci flow and it's geometric applications, Arxiv (2002).

[17] L. Wang, Eigenvalue estimate for the weighted p-Laplace, Annali di Matematica, 191 (2012), $539-550$

[18] L. Wang, Gradient estimates on the weighted p-Laplace heat equation, J. Diff. Equ., 264 (2018), 506-524.

[19] J. Wu, E. Wang and Y. Zheng, First eigenvalue of the p-Laplace operator along the Ricci flow, Ann. Glob. Anal. Geom., 38(1) (2009), 27-55. 
[20] J. Wu, First eigenvalue monotonicity for the p-Laplace operator under the Ricci flow, Acta Mathematica Sinica, English series, (2011), 1591-1598. 